Cremades Andreu, R., García Gil, D., Ramírez Rico, E. y Miraflores Gómez, E. (2016). Acción tutorial en estudiantes de las menciones de educación física y música del grado de maestro en educación primaria. Revista de Investigación Educativa, 34(2), 417-433.

DOI: http://dx.doi.org/10.6018/rie.34.2.237931

\title{
Acción tutorial en estudiantes de las menciones de educación física y música del grado de maestro en educación primaria
}

\section{Tutorial Action of Students of the Specialties of Physical Education and Music in the Degree of Primary Education}

Roberto Cremades Andreu, Desirée García Gil, Elena Ramírez Rico y Emilio Miraflores Gómez

Departamento de Expresión Musical y Corporal, Facultad de Educación, Universidad Complutense de Madrid. España

\section{Resumen}

La puesta en marcha del EEES ha supuesto un cambio en las funciones del profesorado, que debe recurrir a la acción tutorial como medio efectivo para acompañar al alumno en su crecimiento académico, en su inserción laboral y/o en su formación continua. Este trabajo pretende conocer cuáles son las demandas de orientación académica, profesional y personal de 333 estudiantes, 189 hombres (56.8\%) y 144 mujeres (43.2\%), de las menciones de Educación Física y Música del Grado en Educación Primaria de la UCM, durante el curso 2013/14. Para ello, se elaboró un cuestionario y una ficha de seguimiento ad hoc. Los resultados obtenidos resaltaron, además de las diferencias significativas en función de género y curso, que los estudiantes necesitan más asesoramiento sobre Prácticum y TFG, desarrollo de habilidades y competencias, y salidas profesionales. Las conclusiones confirman la importancia de potenciar una acción tutorial continua para el desarrollo integral del individuo.

Palabras clave: acción tutorial; orientación profesional; desarrollo académico; autonomía personal; desarrollo profesional.

Correspondencia: Roberto Cremades Andreu, Facultad de Educación. Universidad Complutense de Madrid. E-mail: rcremades@edu.ucm.es 


\begin{abstract}
The launch of the EHEA has led a change in the functions of the teachers who should draw upon the tutorial action as an effective way to accompany the pupil in his academic growth, when entering the workforce and/or in continuing his training. The purpose of this paper focuses on knowing what the demands of academic, professional and personal orientation are for 333 students, 189 men (56.8\%) and 144 women (43.2\%) who studied specialties of Physical Education and Music from the degree in Primary Education at UCM in the 20132014 academic year. Therefore, a questionnaire and a monitoring sheet were developed ad hoc. The results showed that, in addition to significant differences depending on gender and course, students need more information and advice on issues related to the practicum and the final year dissertation, the development of skills and competences and career opportunities. Conclusions confirm the importance of the promotion of a continuous tutorial action for the integral development of the individual.

Keywords: tutorial action; professional guidance; academic development; personal autonomy; professional development.
\end{abstract}

\title{
Introducción
}

El devenir histórico de la legislación universitaria española, así como de los programas y planes de acción tutorial propuestos, manifiestan que la tutoría en la universidad es uno de los temas que no se ha abordado de manera profunda y satisfactoria, quedando al margen de la función docente (Aguilar et al., 2015; Zabalza, 2014,).

Entre dichos planes y programas tenemos el Primer Plan Nacional de Evaluación de la Calidad de las Universidades (PNECU), establecido por iniciativa del Consejo de Universidades (Real Decreto 1947/1995), vigente entre 1995 y 2000, que impulsó el desarrollo de la evaluación institucional de la calidad en las universidades españolas y elaboró una metodología común para el establecimiento de procesos de evaluación de las titulaciones, de los departamentos y de los servicios. También, puede señalarse el II Plan Calidad de las Universidades (PCU) regulado por el Real Decreto 408/2001, con una vigencia prevista de seis años (aunque fue derogado en 2003) que tuvo el mismo planteamiento del tema e interpretaba el fomento de la calidad en las universidades españolas poniendo especial énfasis en la mejora de los procesos formativos, pero sin referencia alguna a la labor tutorial. En estos planes quedó patente la escasa importancia que se daba a la función tutorial, centrándose, exclusivamente, en la pura transmisión de conocimientos y dejando de lado otras necesidades personales de los estudiantes.

Centrados en el ámbito legislativo universitario, en la Ley Orgánica, 11/1983, de Reforma Universitaria (LRU, 1983), se determinó que la institución educativa superior debería ser entendida como instrumento eficaz de transformación social, al servicio de la libertad, la igualdad y el progreso social para hacer posible una realización más plena de la dignidad humana. En esta normativa, se subrayó el papel que desempeñaban los docentes y discentes para el cumplimiento de esta tarea, pero dejando de lado la tutoría universitaria como parte de la acción docente. Dicha participación activa se consolidó en la Ley Orgánica 6/2001, de Universidades (LOU, 2001), cuando 
en el artículo 46, se determinó que los estudiantes tendrían derecho a orientación e información por parte de la Universidad sobre las actividades de la misma que les afecten, y además, se reconoció su derecho al asesoramiento y a la asistencia por parte de profesores y tutores. Sin embargo, estas propuestas no se llevaron a cabo y sería con la Ley Orgánica 4/2007 (LOMLOU, 2007), que modificaba a la LOU (2001), cuando se indicó que los profesores podrían realizar funciones de tutoría, en la forma que se especificara reglamentariamente. A partir de ese momento, las universidades españolas introdujeron en sus respectivos estatutos la figura tutorial en el desarrollo metodológico de la acción docente.

En paralelo a estos cambios legislativos españoles, se gestaron los procesos de convergencia europea en materia de educación universitaria (Espacio Europeo de Educación Superior - EEES), que implicaron un replanteamiento en el sistema educativo universitario y en la función docente en el que, aparte de las dimensiones académica y profesional, se enfatizaba en la importancia de la acción tutorial y orientación universitaria, atendiendo de este modo, a las demandas de los estudiantes universitarios que no se centran solamente en la asimilación de conocimientos, sino que necesitan adquirir una serie de competencias y destrezas que les permitan profundizar en su formación y/o acceder al mundo laboral (González y Raposo, 2008). A este respecto, Olmos (2014) añade que el profesor universitario debe tener en cuenta "el ámbito social, afectivo y relacional (p. 542)" de sus alumnos para optimizar los procesos de enseñanza-aprendizaje.

Pero, ¿por qué es importante la tutoría y la orientación universitaria? En palabras de García, Asensio, Carballo, García y Guardia (2005), la tutoría universitaria es:

una actividad formativa, encaminada al desarrollo integral (intelectual, profesional y humano) de los estudiantes en el marco de sus estudios universitarios. En definitiva, es una parte de la responsabilidad docente, en la que se establece una interacción particularizada entre el profesor y el estudiante, con el objetivo fundamental de que aquel pueda guiar su aprendizaje, contribuyendo a ir progresivamente adaptándolo a sus circunstancias personales y a su estilo individual de aprender, de modo que cada estudiante logre alcanzar el mayor nivel de dominio posible (pp. 190-191).

En este proceso de tutorización, el estudiante es el protagonista y el docente asume el rol de guía, más allá de su función esencial de dinamizador de conocimientos (Quintanal y Miraflores, 2013). De este modo, las acciones tutoriales personalizadas en el proceso de enseñanza-aprendizaje universitario son imprescindibles (Michavila y García, 2003), pues las necesidades de los estudiantes se agrupan en torno a una triple dimensión:

- Académica: centrada en el rendimiento formativo para la consecución de los objetivos previstos;

- Personal: para un desarrollo integral y mejor conocimiento de sí mismo;

- Profesional: que oriente el proceso formativo hacia la consecución de una profesión y el desarrollo de sus competencias. 
Por lo tanto, el docente universitario no es un mero transmisor de conocimientos y pasa a ser un facilitador del aprendizaje autónomo y un orientador que guía los pasos del estudiante en su proceso de formación (Alcaraz-Salarirche, 2011). Además, el profesor se erige en el dinamizador de la información que recibe el alumno, facilita el aumento de la movilidad estudiantil y laboral que caracteriza a la sociedad postindustrial, al mismo tiempo que participa en el crecimiento académico del individuo (Lapeña, Sauleda y Martínez, 2011; Pantoja, Campoy y Cañas, 2003).

Estas cuestiones que se han potenciado con los cambios producidos por la convergencia europea en materia educativa universitaria, han supuesto un impulso para las metodologías didácticas que consideran a los estudiantes como "aprendices activos, autónomos, estratégicos, reflexivos, cooperativos y responsables, y subrayan planteamientos metodológicos que los implican directamente en el proceso de enseñanza" (Quintanal y Miraflores, 2013, p. 20).

Todo ello supone una visión pedagógica diferente, donde el estudiante deberá implicarse más y con mayor esfuerzo, pero el profesorado tendrá que modificar el enfoque metodológico, que estará centrado en el seguimiento de los estudiantes, en la preparación de sus clases y en la propuesta de nuevas metodologías didácticas, sin olvidar las posibilidades que procuran las recursos online (Rodríguez-Hoyos, Salvador y Salmón, 2015; Sherman y Camilli, 2014).

En este sentido, y teniendo en cuenta las necesidades de los estudiantes universitarios (académica, personal y profesional), han sido varios autores (Álvarez y González, 2008, 2009; Fries-Britt y Snider, 2015; Gairín, Feixas, Guillamón y Quinquer, 2004; García, 2008; Rodríguez, 2004), los que han definido el concepto de acción tutorial universitaria, destacando de sus reflexiones las siguientes ideas:

- La misión del docente universitario va más allá de la pura transmisión de conocimientos y forma parte de su labor educativa.

- El profesorado debe tener en cuenta la necesidad de ayuda a los alumnos durante su proceso educativo en la universidad.

- El profesorado ha de tener conocimiento de sus alumnos, para ayudarles a resolver las dificultades, dudas y problemas.

- La contribución del profesorado al desarrollo integral de la persona en sus dimensiones académica, personal y profesional.

- La tutoría es parte integrante de la función educativa y socializadora del profesor.

En resumen, la acción tutorial viene definida por cuatro parámetros clave (Álvarez, 2012; Feixas, 2001; Henning, Gut y Beam, 2015), que son:

- una guía de incorporación al medio universitario (primeros cursos);

- el apoyo académico (enseñar a estudiar, motivar, informar sobre la asignatura en cuestión, etc.);

- la orientación y la atención a las necesidades personales (fomentar la autonomía y responsabilidad, informar sobre los derechos y obligaciones, encauzar sus demandas e inquietudes, orientarles sobre la manera de resolver dificultades); 
- la orientación profesional (posibles salidas profesionales, información de cursos, seminarios y congresos, bibliografías actualizadas, etc.).

Y para ello, el cometido del profesor universitario, en conexión con las funciones de la acción tutorial expuestas y teniendo en cuenta las aportaciones de diversos autores (Corpas, González, Gutiérrez y Ramírez, 2009; Herrera, 2006), puede resumirse en:

- ayuda a la integración del estudiante en el ámbito universitario.

- planificación e impartición de la docencia de sus asignaturas;

- apoyo en la construcción del conocimiento y del pensamiento crítico;

- favorecimiento del aprendizaje autónomo y experiencial;

- desarrollo de las competencias profesionales pertinentes;

- preparación de los materiales de apoyo al estudiante;

- búsqueda de metodologías favorecedoras del aprendizaje;

- utilización de las tecnologías como herramienta para el aprendizaje del conocimiento;

- búsqueda de modelos de evaluación objetivos y válidos en función de la materia impartida.

Por todo ello, se podría afirmar que la acción tutorial es una de las competencias que, junto a la docencia y la investigación, el profesorado universitario tiene que asumir como parte inseparable e insustituible de su labor docente puesto que significa actuar sobre la formación integral del individuo.

\section{Método}

En esta investigación se ha optado por utilizar un diseño mixto de investigación, combinando el uso de técnicas cuantitativas y cualitativas, para tener una mejor perspectiva del objeto de estudio, que se desarrolló en el curso académico 2013-2014.

\section{Objetivos}

Los objetivos propuestos en esta investigación se concretan en:

a) Examinar cuales son las demandas de orientación académica, profesional y personal de los estudiantes de las menciones de Educación Física y Música.

b) Estudiar la existencia o no de diferencias en las necesidades de orientación del alumnado en función del género y del curso que estudian.

\section{Participantes}

Los participantes en este estudio fueron 333 estudiantes de los cuáles 144 eran mujeres $(43.2 \%)$ y 189 hombres $(56.8 \%)$, con edades comprendidas entre los 19 y 32 años $\left(\mathrm{M}_{\text {edad }}=22.27, \mathrm{DT}_{\text {edad }}=2.43\right)$. Este número de participantes representa el $76.02 \%$ respecto a la muestra total conformada por los estudiantes matriculados en el curso 2013-2014 que fue de 438 (328 mención de Educación Física y 110 mención de Música), cuyas características principales se muestran en la tabla siguiente (ver Tabla 1). 
Tabla 1

Características de la muestra

\begin{tabular}{lccc}
\hline & $(n=333)$ & Frecuencia & Porcentaje (\%) \\
\hline \multirow{3}{*}{ Hombre } & Género & & \\
Mujer & & 189 & 56.8 \\
& & 144 & 43.2 \\
$2^{\underline{0}}$ & Curso & & \\
$3^{\mathbf{o}}$ & & 110 & 33.0 \\
$4^{\underline{0}}$ & & 102 & 30.6 \\
& & 121 & 36.3 \\
Música & Mención & & \\
Educación Física & & 69 & 20.7 \\
\hline
\end{tabular}

Sobre el contexto de esta investigación hay que concretar que los estudiantes tienen la opción de cursar las menciones a partir de segundo curso del Grado en Educación Primaria, razón por la que no hay participantes de primer curso. Añadir también que este estudio incluye a los estudiantes de las menciones de Educación Física y Música puesto que han sido las únicas áreas de especialización docente ofertadas ininterrumpidamente en la Facultad de Educación tanto en las antiguas diplomaturas, como en las menciones establecidas con la entrada en vigor del Plan Bolonia. Si bien, en la actualidad, los estudiantes pueden optar por cursar el resto de menciones (Lengua Extranjera, Audición y Lenguaje y Pedagogía Terapéutica). Además, la presente investigación procede del desarrollo de un proyecto de innovación docente que ha servido para conocer y procurar satisfacer las demandas de información que solicitaron los estudiantes matriculados en dichas menciones, a través de tutorías individuales, grupales y seminarios de trabajo.

\section{Instrumentos de recogida de la información}

Para evaluar las necesidades de información y orientación en el proceso de formación docente de los estudiantes se elaboró un cuestionario ad hoc. Dicho cuestionario se compone de tres bloques. En el primero se solicitan los datos de identificación de los participantes: género, edad, curso y lugar de residencia. En el segundo apartado se solicitan datos académicos: mención que estudian, modalidad de acceso a la universidad, modo de acceso a la mención y si compaginan sus estudios de Grado con otro tipo de estudios. En el último bloque se muestran los ítems que se refieren a las necesidades de información y orientación en torno a las dimensiones académica, profesional y personal, en consonancia con las ideas expuestas por los diferentes autores, que ya se han presentado en la parte teórica de este trabajo. Este instrumento fue evaluado en cuanto 
a sus propiedades psicométricas, de modo que para medir la validez de contenido se utilizó la técnica del juicio de expertos, proceso en el que se consideraron las ideas y las sugerencias que realizaron un total de 7 especialistas en el ámbito educativo universitario. Además, se midió la validez de constructo a través de un análisis factorial con rotación Varimax del que se obtuvieron 3 factores que explicaban el $51.16 \%$ de la varianza total.

En cuanto a la fiabilidad, el resultado obtenido a través de la prueba Alfa de Cronbach fue de .81 por lo que los datos de este estudio muestran un índice aceptable de consistencia interna, considerando como buenos los valores superiores a .70 (Cea, 2012).

Por otra parte, se utilizó una "Ficha de seguimiento de las sesiones de orientación" para complementar la información obtenida a través del cuestionario y para valorar las necesidades concretas que expresaron los estudiantes en las reuniones individuales y grupales llevadas a cabo durante el desarrollo de este estudio.

\section{Tratamiento y análisis de los datos}

Respecto al cuestionario de evaluación inicial se analizaron los datos obtenidos mediante la utilización del programa IBM SPSS Statistics versión 20.0. En primer lugar, se realizó un análisis descriptivo de los datos (media, valores mínimos, máximos y desviación típica). Después se estudió la normalidad de la muestra mediante la prueba de Kolmogorov Smirnov, de la que se obtuvieron puntuaciones de $p$ ๑) .01 rechazándose la hipótesis de normalidad. Así, para el análisis de las dimensiones por género, se utilizó la prueba no paramétrica $U$ de Mann-Whitney para muestras independientes, y para la relación de las dimensiones por curso, la prueba no paramétrica Kruskal-Wallis. Por último, se realizó un análisis de Correlación bivariada por medio del coeficiente de Pearson, para ver si existía asociación entre las dimensiones analizadas.

Por su parte, para procesar la ficha de seguimiento se realizó un análisis de contenido, en cuyo proceso se examina en profundidad lo que los informantes responden a cada una de las cuestiones planteadas sin separarse de las circunstancias y del contexto en el que se realizan (Cáceres, 2008).

\section{Resultados}

\section{Resultados del Cuestionario de evaluación inicial sobre necesidades de informa- ción y orientación}

De este modo, se realizó un análisis estadístico descriptivo de cada uno de los ítems, agrupados en torno a las categorías estudiadas (ver Tabla 2).

Como se observa en la tabla anterior, todos los ítems de las tres dimensiones de análisis (académica, personal y profesional) se sitúan por encima del valor central de la escala. Dentro de los valores obtenidos en la dimensión académica, aquel que obtuvo un valor medio más alto fue el de Practicum y TFG $(\mathrm{M}=4.38$, DT= .79); en la dimensión personal el ítem de desarrollo de habilidades y competencias $(\mathrm{M}=4.07, \mathrm{DT}=.96)$ y en la dimensión profesional el de salidas profesionales $(\mathrm{M}=4.59$, $\mathrm{DT}=.62)$. 
Tabla 2

Estadísticos descriptivos de las necesidades de información y orientación indicadas por los estudiantes

\begin{tabular}{l}
\hline $\begin{array}{l}\text { ¿Sobre qué temas de los que aparecen en la lista siguiente } \\
\text { necesitas información y orientación? }\end{array}$ \\
\hline
\end{tabular}

DIMENSIÓN ACADÉMICA (M=3.57; DT= .58)

1. Plan de estudios y tipo de asignaturas

3.66

0.84

2. Estructura y funcionamiento del Departamento de Expresión Musical y Corporal

$3.49 \quad 0.92$

3. Manejo del servicio UCM en línea (correo, campus virtual, EDUROAM,...)

4. Prácticum y TFG

4.38

0.79

DIMENSIÓN PERSONAL (M= 3.71; DT= .71)

5. Apoyo en el proceso de aprendizaje

6. Técnicas de estudio

7. Talleres de elaboración y presentación de trabajos

8. Búsqueda de fuentes documentales

9. Desarrollo de habilidades y competencias

10. Resolución de conflictos personales (falta de motivación, autoestima,...)

\section{DIMENSIÓN PROFESIONAL (M=4.22; DT= .58)}

11. Asesoramiento sobre elaboración de Curriculum Vitae

12. Estrategias para la entrevista de trabajo

13. Dimensión profesional de las asignaturas de las menciones

15. Información sobre intercambio internacional a nivel profesional

A continuación, en el análisis realizado de cada uno de los ítems que componen la dimensión académica en función del género, los resultados mostraron diferencias estadísticamente significativas en el ítem 1. Plan de estudios y tipo de asignaturas (U= 11135.000; $Z=-3.055 ; p=.002)$; en el ítem 2. Estructura y funcionamiento del Departamento de Expresión Musical y Corporal $(\mathrm{U}=11685.500 ; \mathrm{Z}=-2.241 ; p=.025)$ y en el ítem 4. Prácticum y TFG ( $\mathrm{U}=10658.000 ; \mathrm{Z}=-3.701 ; p=.000)$, donde son siempre las mujeres las que presentan un rango promedio mayor que los hombres (ver Tabla 3). 
Tabla 3

Análisis de comparación a través de la prueba U de Mann-Whitney, de la dimensión académica en función de la variable género

\begin{tabular}{clccccc}
\hline ÍTEMS & Género & $\begin{array}{c}\text { Rango } \\
\text { Promedio }\end{array}$ & $\begin{array}{c}\text { Suma de } \\
\text { rangos }\end{array}$ & $\begin{array}{c}\text { U de } \\
\text { Mann-Whitney }\end{array}$ & Z & $p$ \\
\hline \multirow{2}{*}{ I.1 } & Hombre & 153.92 & 29090.00 & 11135.000 & -3.055 & $.002^{* *}$ \\
& Mujer & 184.17 & 26521.00 & & & \\
I.2 & Hombre & 156.83 & 29640.50 & 11685.500 & -2.241 & $.025^{*}$ \\
& Mujer & 179.28 & 25637.50 & & & \\
I.3 & Hombre & 167.60 & 31509.50 & 13328.500 & -.250 & .803 \\
& Mujer & 165.06 & 23768.50 & & & \\
I.4 & Hombre & 151.19 & 28424.00 & 10658.000 & -3.701 & $.000^{* * *}$ \\
\hline
\end{tabular}

${ }^{*} p<.05 ;{ }^{* *} p<.01 ;{ }^{* * *} p<.001$

En la tabla 4, respecto a la dimensión personal, se observan diferencias estadísticamente significativas en el ítem 5. Apoyo en el proceso de aprendizaje ( $U=11184.500 ; Z=-3.043$; $p=.002)$; en el ítem 6. Técnicas de estudio $(\mathrm{U}=10818.500 ; \mathrm{Z}=-3.296 ; p=.001)$; en el ítem 7 . Talleres de elaboración y presentación de trabajos ( $\mathrm{U}=11044.000 ; \mathrm{Z}=-3.161 ; p=.002)$ y en el ítem 8. Búsqueda de fuentes documentales ( $\mathrm{U}=11741.500 ; \mathrm{Z}=-2.268 ; p=.023)$, donde son siempre las mujeres las que presentan un rango promedio superior al de los hombres.

Tabla 4

Análisis de comparación a través de la prueba $U$ de Mann-Whitney, de las dimensión personal en función de la variable género

\begin{tabular}{|c|c|c|c|c|c|c|}
\hline ÍTEMS & Género & $\begin{array}{c}\text { Rango } \\
\text { Promedio }\end{array}$ & Suma de rangos & $\begin{array}{c}\text { U de } \\
\text { Mann-Whitney }\end{array}$ & $\mathbf{Z}$ & $p$ \\
\hline \multirow{2}{*}{ I.5 } & Hombre & 154.18 & 29139.50 & \multirow{2}{*}{11184.500} & \multirow{2}{*}{-3.043} & \multirow{2}{*}{$.002^{* *}$} \\
\hline & Mujer & 183.83 & 26471.50 & & & \\
\hline \multirow{2}{*}{ I.6 } & Hombre & 152.24 & 28773.50 & \multirow{2}{*}{10818.500} & \multirow{2}{*}{-3.296} & \multirow{2}{*}{$.001^{* *}$} \\
\hline & Mujer & 185.35 & 26504.50 & & & \\
\hline \multirow{2}{*}{ I.7 } & Hombre & 153.43 & 28999.00 & \multirow{2}{*}{11044.000} & \multirow{2}{*}{-3.161} & \multirow{2}{*}{$.002^{* *}$} \\
\hline & Mujer & 184.81 & 26612.00 & & & \\
\hline \multirow{2}{*}{ I. 8} & Hombre & 157.12 & 30153.00 & \multirow{2}{*}{11741.500} & \multirow{2}{*}{-2.268} & \multirow{2}{*}{$.023^{*}$} \\
\hline & Mujer & 179.96 & 25458.00 & & & \\
\hline \multirow{2}{*}{ I.9 } & Hombre & 159.54 & 30153.00 & \multirow{2}{*}{12198.000} & \multirow{2}{*}{-1.773} & \multirow{2}{*}{.076} \\
\hline & Mujer & 176.79 & 25453.00 & & & \\
\hline \multirow{2}{*}{1.10} & Hombre & 161.26 & 30478.50 & \multirow{2}{*}{12523.500} & \multirow{2}{*}{-1.305} & \multirow{2}{*}{.192} \\
\hline & Mujer & 174.53 & 25132.50 & & & \\
\hline
\end{tabular}


En la dimensión profesional, las diferencias estadísticamente significativas se encuentran en los cinco ítems que la componen, esto es en el ítem 11. Asesoramiento sobre elaboración del curriculum vitae $(\mathrm{U}=11128.000 ; \mathrm{Z}=-2.984 ; p=.003)$; ítem 12. Estrategias para la entrevista de trabajo $(\mathrm{U}=10583.000 ; \mathrm{Z}=-3.671 ; p=.003)$; ítem 13. Dimensión profesional de las asignaturas de las menciones $(\mathrm{U}=11353.000 ; \mathrm{Z}=-2.816 ; p=.005)$; ítem 14 . Salidas profesionales $(\mathrm{U}=11021.000 ; \mathrm{Z}=-3.309 ; p=.001) \mathrm{y}$ el ítem 15. Información sobre intercambio internacional a nivel profesional $(\mathrm{U}=11099.00 ; \mathrm{Z}=-3.040 ; p=.002)$. Al igual que en los apartados anteriores, son siempre las mujeres las que recogen una mayor valoración a tratar los aspectos contemplados en los ítems (ver Tabla 5).

Tabla 5

Análisis de comparación a través de la prueba $U$ de Mann-Whitney, de la dimensión profesional en función de la variable género

\begin{tabular}{clccccc}
\hline ÍTEMS & Género & $\begin{array}{c}\text { Rango } \\
\text { Promedio }\end{array}$ & $\begin{array}{c}\text { Suma de } \\
\text { rangos }\end{array}$ & $\begin{array}{c}\text { U de } \\
\text { Mann-Whitney }\end{array}$ & Z & $p$ \\
\hline \multirow{2}{*}{ I.11 } & Hombre & 153.88 & 29083.00 & 11128.000 & -2.984 & $.003^{* *}$ \\
& Mujer & 184.22 & 26528.00 & & & \\
\multirow{2}{*}{ I.12 } & Hombre & 150.99 & 28538.00 & & & \\
& Mujer & 188.01 & 27073.00 & 10583.000 & -3.671 & $.000^{* * *}$ \\
\multirow{2}{*}{ I.13 } & Hombre & 155.07 & 29308.00 & & & \\
& Mujer & 182.66 & 26303.00 & 11353.000 & -2.816 & $.005^{* *}$ \\
I.14 & Hombre & 153.31 & 28976.00 & & & \\
& Mujer & 184.97 & 26635.00 & 11021.000 & -3.309 & $.001^{* *}$ \\
I.15 & Hombre & 153.72 & 29054.00 & & & \\
& Mujer & 184.42 & 26557.00 & 11099.00 & -3.040 & $.002^{* *}$ \\
\hline
\end{tabular}

${ }^{* *} p<.01 ;{ }^{* * *} p<.001$

A continuación, se llevaron a cabo los análisis de comparación de los ítems de cada una de las dimensiones por curso, de los cuales se presentan únicamente los resultados de la académica y personal, ya que en la profesional no se hallaron diferencias estadísticamente significativas. Así, en la dimensión académica, los resultados ofrecen diferencias significativas en el ítem 1. Plan de estudios y tipo de asignaturas $\left(\mathrm{X}^{2}=8.106\right.$; $p=.017)$. Estas diferencias indican que el alumnado de $4^{\underline{0}}$ curso valora más que los de $2^{\underline{o}}$ profundizar en la información sobre las características particulares de los estudios que están realizando (ver Tabla 6).

En cuanto a los análisis de la dimensión personal, los resultados de la tabla 7 destacan diferencias significativas en el ítem 6. Técnicas de estudio $\left(X^{2}=10.156 ; p=.006\right)$ en que el alumnado de $3^{\circ}$ curso valora más que los de $4^{\circ}$ profundizar en este tema. Por su parte, en el ítem 8. Búsqueda de fuentes documentales $\left(X^{2}=8.646 ; p=.013\right)$, es el alumnado de $3^{\circ}$ y $4^{\underline{0}}$ curso el que más valora el desarrollar esta temática en las sesiones de orientación en relación con el alumnado de $2^{\circ}$ curso (ver Tabla 7). 
Tabla 6

Análisis de comparación a través de la prueba Kruskal-Wallis, de la dimensión académica en función de la variable curso

\begin{tabular}{|c|c|c|c|c|c|}
\hline ITEMS & Curso & $\begin{array}{c}\text { Rango } \\
\text { Promedio }\end{array}$ & $X^{2}$ & $p$ & $\begin{array}{c}\text { Comparaciones U de } \\
\text { Mann-Whitney }\end{array}$ \\
\hline \multirow{3}{*}{ I.1 } & $2^{\mathrm{O}}$ & 149.22 & \multirow{3}{*}{8.106} & \multirow{3}{*}{$.017^{*}$} & \multirow{3}{*}{$4^{\mathrm{O}}>2^{\mathrm{O}}$} \\
\hline & $3^{\mathrm{o}}$ & 167.43 & & & \\
\hline & $4^{\mathrm{o}}$ & 182.80 & & & \\
\hline \multirow{3}{*}{ I. 2} & $2^{\mathrm{o}}$ & 162.93 & \multirow{3}{*}{1.765} & \multirow{3}{*}{.414} & \\
\hline & $3^{\mathrm{o}}$ & 176.35 & & & \\
\hline & $4^{\mathrm{o}}$ & 161.41 & & & \\
\hline \multirow{3}{*}{ I. 3} & $2^{\mathrm{o}}$ & 152.95 & \multirow{3}{*}{5.841} & \multirow{3}{*}{.054} & \\
\hline & $3^{\mathrm{o}}$ & 183.37 & & & \\
\hline & $4^{\mathrm{o}}$ & 164.48 & & & \\
\hline \multirow{3}{*}{ I. 4} & $2^{\mathrm{o}}$ & 153.08 & \multirow{3}{*}{5.588} & \multirow{3}{*}{.061} & \\
\hline & $3^{\mathrm{o}}$ & 165.00 & & & \\
\hline & $4^{\mathrm{o}}$ & 179.86 & & & \\
\hline
\end{tabular}

${ }^{*} p<.05$

Tabla 7

Análisis de comparación a través de la prueba Kruskal-Wallis, de la dimensión personal en función de la variable curso

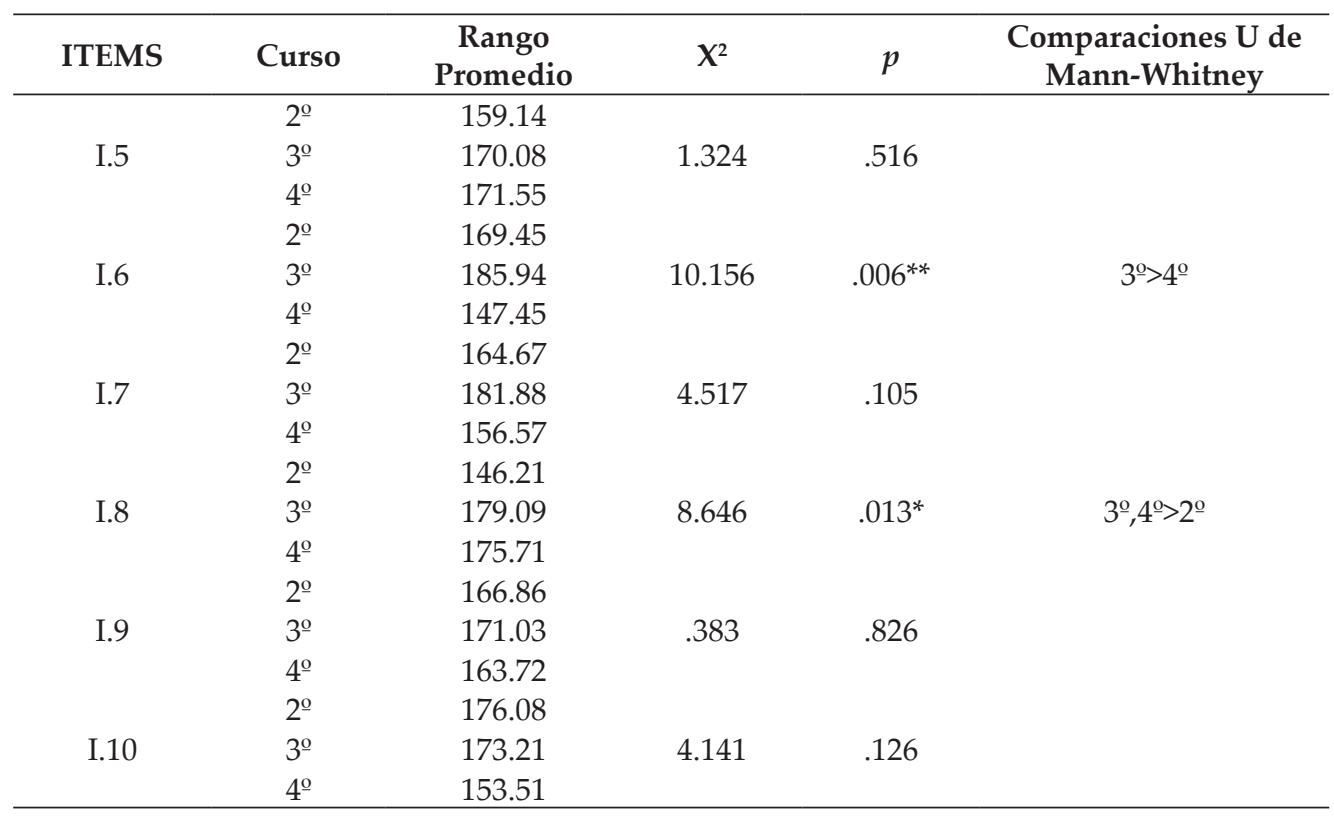

${ }^{*} p<.05 ;{ }^{* *} p<.01$ 
Por último, la Tabla 8 ofrece los resultados obtenidos en el análisis de correlación entre la dimensión académica, personal y profesional (ver Tabla 8).

Tabla 8

Análisis de correlación de Pearson entre la dimensión académica, personal y profesional

\begin{tabular}{lcc}
\hline & Dimensión académica & Dimensión personal \\
\hline Dimensión personal & $.319^{* * *}$ & \\
Dimensión profesional & $.327^{* * *}$ & $.384^{* * *}$ \\
${ }^{* * * *} p<.001$ & &
\end{tabular}

Como se muestra en la tabla anterior existe una correlación significativa débil para las dimensiones académica-personal ( $r=.319 ; p=.000)$; dimensiones académica-profesional $(r=.327 ; p=.000) ; \mathrm{y}$ dimensiones personal-profesional $(r=.384 ; p=.000)$. La relación entre las dimensiones personal-profesional es donde se encuentra la correlación más fuerte, a pesar de que todas las correlaciones son débiles.

\section{Resultados de la ficha de seguimiento de las sesiones de orientación}

El análisis de contenido extraído de las fichas de seguimiento reveló, en relación con la dimensión académica, que el alumnado de ambas menciones vio truncadas sus expectativas sobre las mismas, al subrayar su descontento con la formación recibida, puesto que, según ellos, eran insufici[entes] [el] número de asignaturas que las conformaban. A pesar de este primer descontento, resultó llamativo el interés de los estudiantes en profundizar en los conocimientos específicos propios de cada área, por lo que demandaron información sobre el modo de poder realizar una formación complementaría tanto en el ámbito de la Educación Física, como de la Música, que sirviera para mejorar su preparación. Sin embargo, los estudiantes valoraron positivamente tanto las materias que estaban cursando en ese momento, que llegaron a señalar como imprescindibles en el desarrollo integral de todo individuo, como por el profesorado que las impartía. En algunos casos, los estudiantes se interesaron por la realización de actividades formativas que se ofertaban en la Facultad de Educación.

En referencia a la dimensión académica, los participantes mostraron un amplio desconocimiento sobre las pautas de elaboración de su Trabajo Fin de Grado (TFG), aún estando disponibles en la Web de la Universidad Complutense y contando éstos con un tutor individual para la realización del mismo. Además, también manifestaron sus dudas en cuanto a la elección de la temática de sus trabajos, así como en lo relativo a la búsqueda de referencias bibliográficas específicas de cada área.

Por último, en lo referente a las cuestiones profesionales se interesaron por la continuación de sus estudios una vez acabado el Grado en Educación Primaria, centrando sus necesidades en el acceso a estudios de Posgrado, tanto en Másteres 
de investigación como profesionalizantes, afines a sus titulaciones, no solo en universidades españolas sino también extranjeras. Igualmente, solicitaron información sobre cuáles eran los procedimientos para acceder a la docencia en centros concertados y públicos.

\section{Discusión y conclusiones}

En función de los objetivos propuestos en esta investigación, los resultados obtenidos muestran las necesidades de orientación de los estudiantes de las menciones de Educación Física y Música desde la dimensión académica, profesional y personal, así como las cuestiones que más les preocupan en el desarrollo de sus estudios. Concretamente, en relación con la dimensión académica los participantes señalan en mayor medida, tanto en el cuestionario como en las fichas de orientación, que necesitan profundizar en los aspectos relativos al Prácticum y el TFG, a pesar de disponer de toda la información necesaria en la Web del centro, circunstancia que está relacionada con la importancia que tienen estas materias, que además, sirven para valorar la adquisición de las competencias docentes desarrolladas a lo largo del Grado. Sobre la dimensión personal, los estudiantes solicitan información sobre la adquisición de habilidades y competencias personales centradas en el desarrollo de su actividad como futuros docentes. Esta elección guarda una estrecha relación con la preocupación que muestran los estudiantes en su propio desempeño en el aula en el período de prácticas, y pone de manifiesto nuevamente, la importancia que tiene esta materia. En cuanto a la dimensión profesional, el interés del alumnado se focaliza en la orientación sobre salidas profesionales, aspecto que reveló un cierto desconocimiento de las posibilidades laborales de las menciones. Así, estos datos indican que se debería procurar ampliar la visión sobre la función docente universitaria más allá de la mera transmisión de conocimientos (Álvarez, 2012; Gairín et al., 2004), ratificando en consecuencia, la necesidad de apoyar y orientar a los estudiantes en su proceso de formación académica y profesional (Herrera, 2006; Rodríguez-Hoyos et al., 2015), y por ende, personal (Álvarez y González, 2008; Feixas, 2001).

Es necesario señalar también, que los participantes en este estudio no demandan una ayuda paternalista y/o de una resolución inmediata del problema, exclusivamente, sino un apoyo y orientación que procure procesos autónomos de aprendizaje que les sirvan en su futuro desempeño profesional (autor y autor, 2013). Asimismo, señalan diferentes problemas que se les habían planteado en su vida académica, destacando, entre otros, los siguientes:

- el veto que supone la elección de una mención, no pudiendo acceder a otras áreas;

- el insuficiente número de asignaturas que se imparten en la mención para una formación adecuada;

- la situación general de las asignaturas de la carrera, que les ofrecen más carga lectiva teórica que práctica. 
Igualmente, los estudiantes indican que es necesario tener un profesor que sea el referente de cada mención. Esto es, consideran imprescindible la existencia de una persona cercana que resuelva las dudas que no plantean en las tutorías ordinarias, por falta de confianza o timidez.

En relación con los resultados obtenidos por género, son las mujeres quienes muestran un mayor interés en informarse sobre aspectos relativos a la dimensión académica (Plan de estudios y tipo de asignaturas, estructura y funcionamiento del departamento de Expresión Musical y Corporal, y Prácticum y TFG); la dimensión personal (Apoyo en el proceso de aprendizaje, técnicas de estudio, talleres de elaboración y presentación de trabajos y Búsqueda de fuentes documentales), y en la dimensión profesional (Asesoramiento sobre elaboración del curriculum vitae, estrategias para la entrevista de trabajo, dimensión profesional de las asignaturas de las menciones, salidas profesionales e información sobre intercambio internacional a nivel profesional). Este hecho puede estar relacionado con el mayor interés en su proceso de aprendizaje que poseen las alumnas frente a los alumnos (Castejón, 2014), así como, a su mejor rendimiento académico (Echavarri, Godoy y Olaz, 2007).

Por cursos, los datos obtenidos revelan, en cuanto a la dimensión académica, que los estudiantes de $4^{\mathrm{o}}$ valoran más que los de $2^{\mathrm{o}}$ obtener información sobre el plan de estudios y el tipo de asignaturas, circunstancia que se podría explicar en el hecho de que muchos de ellos estaban interesados en continuar su formación y querían saber qué posibilidades les ofrecía su carrera académica. Sobre la dimensión personal, los resultados indican que el alumnado de $3^{0}$ curso solicita más apoyo sobre técnicas de estudio que los de $4^{\circ}$ curso, puesto que estos últimos se encuentran finalizando sus estudios de Grado y tienen un mayor nivel de competencias en la realización de trabajos académicos. En relación con la búsqueda de fuentes documentales los datos señalan que los alumnos de $3^{\circ}$ y $4^{\circ}$ son los que presentan una manifiesta necesidad de información sobre este aspecto, debido a que se encuentran perfilando detalles para desarrollar su TFG.

Además, los resultados que arrojó el análisis de correlación de Pearson muestran claramente, aunque en valores débiles, que existe una relación entre las dimensiones académica-personal, académica-profesional y personal-profesional. Estos datos, aunque con ciertas limitaciones, ponen de relieve la importancia de incluir en la acción tutorial orientaciones estructuradas en torno a los aspectos académicos, personales y profesionales, en consonancia con los resultados obtenidos en otros estudios (Michavila y García, 2003).

En definitiva, el estudio presentado enfatiza la importancia que posee la acción tutorial y el asesoramiento académico, personal y profesional para los participantes en este trabajo, al mismo tiempo que pone de manifiesto la necesidad de procurar un cambio en el modo de entender el proceso de enseñanza-aprendizaje universitario, que debería centrarse en nuevas metodologías didácticas (Sherman y Camilli, 2014), en un estilo individual y personal de aprender (García et al., 2005), en una aplicación eficiente de la acción tutorial universitaria (Michavila y García, 2003) y en el uso de la tecnología como apoyo didáctico y autónomo en el aprendizaje (Zabalza, 2014). De este modo, la función de la tutoría debe procurar un traspaso efectivo de información, en la que los docentes se conviertan en verdaderos tutores que señalen las vías de 
actuación, dejando que sean los propios intervinientes quienes solventen sus dudas, dándoles herramientas de actuación, es decir, procurar ofrecer un aprendizaje guiado de los diferentes y múltiples ámbitos de interés de los estudiantes.

\section{Referencias}

Aguilar, J. M., Alías, A., Álvarez, J., Fernández, J. M., Pérez, E.R., y Hernández, A. I. (2015). Necesidades de formación del profesor universitario en competencias relacionadas con la acción tutorial. Revista de Docencia Universitaria, REDU, 13(3), 357-378.

Alcaraz-Salarirche, N (2011). An Action Research Process on University Tutorial Sessions with Small Groups: Presentational Tutorial Sessions and Online Communication. Educational action research, 19(4), 549-565. doi:10.1080/09650792.2011.625713

Álvarez, P. R. (2012). Tutoría universitaria inclusiva. Guía de buenas prácticas para la orientación de estudiantes con necesidades educativas específicas. Madrid: Narcea.

Álvarez, P. y González, M. (2008). Los planes de tutoría en la Universidad: una guía para su implantación. Tenerife: Servicio de Publicaciones de la Universidad de La Laguna.

Álvarez, P. y González, M. (2009). Modelo comprensivo para la institucionalización de la orientación y la tutoría en la enseñanza universitaria. Revista Curriculum, 22, 73-96. Recuperado de http://revistaq.webs.ull.es/ANTERIORES/numero22/alvarez.pdf

Cáceres, P. (2008). Análisis cualitativo de contenido: Una alternativa metodológica alcanzable. Psicoperspectivas. Individuo y Sociedad, 2(1), 53-82. Recuperado de http:// psicoperspectivas.cl/index.php/psicoperspectivas/article/download/3/3

Castejón, J. L. (2014). Aprendizaje y rendimiento académico. Alicante: Editorial Club Universitario.

Cea, M. A. (2012). Fundamentos y aplicaciones en metodología cuantitativa. Madrid: Editorial Síntesis.

Corpas, C., González, I., Gutiérrez, M. P., y Ramírez, A. (2009). La formación en competencias del profesorado universitario: resultados de una experiencia. En BozaCarreño, B., Méndez-Garrido, J. M., Monescillo-Palomo, M., Toscano-Cruz, M., Aguaded-Gómez, M., Ávila Fernández, J.A., Tello Díaz, M., \& Salas Tenorio, M. (Coords.), Educación, investigación y desarrollo social. Actas del XIV Congreso Nacional de modelos de Investigación Educativa (AIDIPE) (591-598). Huelva: Universidad de Huelva.

Echavarri, M., Godoy, J. C., y Olaz, F. (2007). Diferencias de género en habilidades cognitivas y rendimiento académico en estudiantes universitarios. Universitas Psychologica, 6(2), 319-329. Recuperado de http://pepsic.bvsalud.org/scielo.php?pid=S165792672007000200011\&script=sci_arttext

Feixas, M. (2001). El desarrollo profesional del profesor universitario como docente. (Tesis inédita de doctorado). Barcelona, Universidad Autónoma de Barcelona

Fries-Britt, S., y Snider, J. (2015). Mentoring Outside the Line: The Importance of Authenticity, Transparency, and Vulnerability in Effective Mentoring Relationships. New Directions for Higher Education, 171, 3-11. doi:10.1002/he.20137

Gairín, J., Feixas, M., Guillamón, C., y Quinquer, D. (2004). La tutoría académica en el escenario europeo de Educación Superior. Revista Interuniversitaria de Formación del Profesorado, 18(1), 66-77. Recuperado de http://dialnet.unirioja.es/descarga/articulo/1057097.pdf 
García, N. (2008). La función tutorial de la Universidad en el actual contexto de la Educación Superior. Revista interuniversitaria de formación del profesorado, 22(1), 21-48. Recuperado de http://www.redalyc.org/articulo.oa?id=27413170002

García, N., Asensio, I., Carballo, R., García, M., y Guardia, S. (2005). La tutoría universitaria ante el proceso de armonización europea. Revista de Educación, 337, 189-210. Recuperado de http://www.revistaeducacion.mec.es/re337/re337_10.pdf

González, M. y Raposo, M. (2008). Necesidades formativas del profesorado universitario en el contexto de la convergencia europea. Revista de Investigación Educativa, 26(2), 285-306.

Henning, J. E., Gut, D., y Beam, P. (2015). Designing and Implementing a Mentoring Program to Support Clinically-Based Teacher Education. Teacher Educator, 50(2), 145-162. doi:10.1080/08878730.2015.1011046

Herrera, L. (2006). El futuro de la psicopedagogía en el marco de la Convergencia Europea de Educación Superior. En M.A. Gallardo, J.A. Fuentes, L. Herrera, S. Rodríguez, G. Rojas, D. Seijo, J.L. Villena y A.M. Fernández (Coords.), I Jornadas de Psicopedagogía: Evaluación e Intervención en Contextos Educativos (pp. 1-13). Granada: Proyecto de Innovación Docente "Plan de Mejora y Evaluación del Prácticum de Psicopedagogía en Melilla".

Lapeña, C., Sauleda, N., y Martínez, A. (2011). Los programas institucionales de acción tutorial: una experiencia desarrollada en la Universidad de Alicante. Revista de Investigación Educativa, 29(2), 341-361.

Ley Orgánica 11/1983, de 25 de agosto, de Reforma Universitaria (BOE no 209 de 1 de septiembre de 1983).

Ley Orgánica 4/2007, de 12 de abril, por la que se modifica la Ley Orgánica 6/2001, de 21 de diciembre, de Universidades (BOE $\mathrm{n}^{\circ}$. 89 de 13 de abril de 2007).

Ley Orgánica 6/2001, de 21 de diciembre, de Universidades (BOE nº 307 de 24 de diciembre de 2001).

Michavila, F. y García, J. (Eds.) (2003). La tutoría y los nuevos modos de aprendizaje en la universidad. Madrid: Consejería de Educación de la CAM y Cátedra Unesco de Gestión y Política Universitaria. Universidad Politécnica de Madrid.

Olmos, P. (2014). Competencias básicas y procesos perceptivos: factores clave en la formación y orientación de los jóvenes en riesgo de exclusión educativa y sociolaboral. Revista de Investigación Educativa, 32(2), 531-546.

Pantoja, A., Campoy, T. J., y Cañas, A. (2003). Un estudio multidimensional sobre la orientación y la acción tutorial en las diferentes etapas del sistema educativo. Revista de Investigación Educativa, 21 (1), 67-91.

Quintanal, J. y Miraflores, E. (2013). Un modelo de tutoría en la universidad del siglo XXI. Madrid: CCS.

Real Decreto 1947/1995, de 1 de diciembre, por el que se establece el Plan Nacional de Evaluación de la Calidad de las Universidades (BOE no. 294 de 9 de diciembre de 1995).

Real Decreto 408/2001, de 20 de abril, por el que se establece el II Plan de la Calidad de las Universidades (BOE nํ. 96 de 21 de abril de 2001).

Rodríguez, S. (Coord.) (2004). Manual de tutoría universitaria. Recursos para la acción. Barcelona: Octaedro. 
Rodríguez-Hoyos, C., Salvador, A. C., y Salmón, I. H. (2015). La tutoría académica en la educación superior. Una investigación a partir de entrevistas y grupos de discusión en la Universidad de Cantabria (España). Revista Complutense de Educación, 26(2), 467481. Recuperado de http://revistas.ucm.es/index.php/RCED/article/view/43745/45519

Sherman, S. y Camilli, G. (2014). Evaluation of an Online Mentoring Program. Teacher Education Quarterly, 41(2), 107-119. Recuperado de http://files.eric.ed.gov/fulltext/ EJ1078644.pdf

Zabalza, M. A. (2014). Formación docente del profesorado universitario. El difícil tránsito a los enfoques institucionales. Revista española de pedagogía, 72(257), 39-54.

Fecha de recepción: 27 de septiembre de 2015.

Fecha de revisión: 27 de septiembre de 2015.

Fecha de aceptación: 29 de marzo de 2016. 
